\title{
CONSTRUÇÃO COLETIVA DE ESTRATÉGIAS PARA UM PROGRAMA DE EDUCAÇÃO PERMANENTE EM TRANSPLANTE HEPÁTICO
}

\section{Collective construction of strategies for a program of continuing education in liver transplantation}

\section{Construcción colectiva de estrategias para un programa de educación permanente en transplante hepático}

\author{
Elisa Borges Kuze ${ }^{1}$ D, Lúcia Nazareth Amante ${ }^{2 *}$ (D), Juliana Balbinot Reis Girondi²(D), \\ Luciara Fabiane Sebold² ${ }^{\mathbb{D}}$, Nádia Chiodelli Sallum² ${ }^{2}$, Neide da Silva Knihs ${ }^{3}$ iD
}

RESUMO: Objetivo: Identificar, junto à equipe de enfermagem, estratégias educativas para a composição de um plano de ações educacionais em transplante hepático para a enfermagem do centro cirúrgico. Método: Pesquisa exploratória, descritiva, qualitativa, realizada em um hospital escola da região sul do país, aprovada pelo comitê de ética em pesquisas envolvendo seres humanos. Resultados: Participaram 16 integrantes da equipe de transplante hepático, sendo que na análise de conteúdo emergiram três categorias: conhecimento de todo o processo de transplante hepático; apropriação dos cuidados de enfermagem perioperatória em transplante hepático; e integração e qualificação da equipe interdisciplinar. Conclusão: Deseja-se que esta pesquisa sirva de auxílio para outros centros transplantadores, sendo significativo o aprofundamento no que concerne à implantação do serviço com embasamento em um referencial metodológico para alicerçar a prática, configurando-o como um instrumento científico para o desenvolvimento das atividades e a formação de programas de educação permanente, de acordo com as necessidades das equipes que atuam com essa realidade.

Palavras-chave: Transplante hepático. Educação continuada. Capacitação em serviço. Enfermagem de centro cirúrgico.

ABSTRACT: Objective: Find out educational strategies, along with the nursing team, to be able for elaborate a plan of educational actions in liver transplantation for surgical center nursing. Method: Exploratory, descriptive, qualitative research, carried out in a school hospital in southern Brazil, approved by the human research ethics committee. Results: 16 members of the liver transplantation team took part in this research, and by analyzing the context, three categories emerged: knowledge of the whole liver transplantation process; appropriation of perioperative nursing care in liver transplantation; and integration and qualification of the interdisciplinary team. Conclusion: We expect that this research will help other transplantation centers, as it is significantly deep regarding the implementation of services. It is based on a methodological framework to support the practice, configuring it as a scientific instrument for the development of activities and the formation of continuing education programs, according to the needs of the teams working with this reality. Keywords: Liver transplantation. Continuing education. In-service training. Surgery center nursing.

RESUMEN: Objetivo: Identificar junto con el equipo de enfermería las estrategias educativas para la composición de un plan de acciones educacionales en el trasplante hepático para la enfermería del centro quirúrgico. Método: Investigación exploratoria, descriptiva y cualitativa realizada en un hospital escuela de la región sur del país y aprobada por el comité de ética en investigaciones con seres humanos. Resultados: Participaron 16 integrantes del equipo de trasplante hepático, siendo que en el análisis del contenido surgieron tres categorías: conocimiento de todo el proceso de trasplante hepático, apropiación de los cuidados de la enfermería perioperatoria en el trasplante hepático e integración y calificación del equipo interdisciplinario. Conclusión: Se desea que esta investigación sirva de auxilio para otros centros de trasplantes, siendo significativo el ahondamiento en relación a la implantación del

'Enfermeira do Hospital Universitário, filial da Empresa Brasileira de Serviços Hospitalares; mestre profissional em Gestão do Cuidado em Enfermagem pela Universidade Federal de Santa Catarina (UFSC) Florianópolis (SC), Brasil.

${ }^{2}$ Docente da UFSC; doutora em Enfermagem pela UFSC - Florianópolis (SC), Brasil.

${ }^{3}$ Docente da UFSC; doutora em Enfermagem pela Universidade Federal de São Paulo (UNIFESP) - São Paulo (SP), Brasil.

*Autor correspondente: luciamante@gmail.com

Recebido: 11/09/2017 - Aprovado: 14/04/2018

DOI: $10.5327 / 21414-4425201800030002$ 
servicio con fundamento en un referente metodológico para cimentar la práctica, configurándola como un instrumento científico para el desarrollo de las actividades y la formación de programas de educación permanente y de acuerdo con las necesidades de los equipos que actúan con esa realidad. Palabras clave: Transplante de hígado. Educación continuada. Capacitación em servicio. Enfermería de quirófano.

\section{INTRODUÇÃO}

A Política Nacional de Educação Permanente em Saúde, em vigor desde 2004, foi criada como estratégia para transformação da prática ${ }^{1}$. As boas práticas em saúde requerem um sistema organizacional relacionado à educação em serviço e educação continuada. Nessa perspectiva, a educação permanente está ligada ao processo, ao ensino e à gestão do cuidado, devendo ser assumida como uma prática diária, de modo reflexivo ${ }^{2}$.

Por conseguinte, a educação permanente configura-se como uma ferramenta para atualização do conhecimento teórico-prático dos profissionais de enfermagem que atuam no serviço de transplante, bem como para a implantação de intervenções atualizadas direcionadas às principais necessidades do paciente em transplante hepático. Esta é compreendida como a constante busca pelo aprender, como uma das ações que possibilita o desenvolvimento do processo de mudança e que visa à qualificação profissional da enfermagem e, consequentemente, à realização da prática profissional competente, consciente e responsável ${ }^{3}$.

A equipe de enfermagem que atua no processo de transplante de órgãos e tecidos necessita de um amplo conhecimento técnico e científico, uma vez que o contexto geral do procedimento é complexo e está em constante atualização. Nesse cenário, cabe ao enfermeiro participar da organização e do desenvolvimento de um programa de transplante efetivo e eficiente, para promover um cuidado de qualidade, por meio de tecnologias, logísticas e recursos humanos, para o aperfeiçoamento das atividades de coordenação, assistência, educação permanente e pesquisa, relacionadas ao transplante de órgãos e tecidos ${ }^{4}$.

O preparo da equipe de saúde do centro cirúrgico (CC), especificamente considerando o período intraoperatório para o processo de transplante hepático, promove a segurança do paciente, pois evita a sua exposição ao risco de incidentes e eventos adversos durante a cirurgia. nesse sentido, há necessidade de criar programas de capacitação para os profissionais de enfermagem, lançando métodos educativos dinâmicos e contextualizados.

Sendo assim, o problema deste estudo é: Quais estratégias educativas podem compor um programa de educação permanente sobre cuidados e rotinas no período intraoperatório em transplante hepático para os profissionais de enfermagem?

\section{OBJETIVO}

Identificar, junto à equipe de enfermagem, estratégias educativas para a composição de um plano de ações educacionais em transplante hepático para a enfermagem de CC.

\section{MÉTODO}

Trata-se de uma pesquisa exploratória, descritiva, de abordagem qualitativa ${ }^{5}$, desenvolvida no CC de um hospital escola do sul do Brasil, no período de novembro a dezembro de 2016 .

Na referida instituição, existe o serviço de transplante hepático, implantado em novembro de 2011, com média de $1,22 \%$ transplantes ao mês, que até junho de 2017 efetivou 85 transplantes e teve 106 pacientes inscritos no programa ${ }^{6}$.

Para efetivação do serviço, existe uma rotina de ações e cuidados desenvolvidos por toda a equipe de saúde, especialmente pela enfermagem, que se configura o objeto do estudo que se apresenta. a rotina de trabalho atende os aspectos de: organização da equipe de transplante hepático; preparação da sala cirúrgica para realização do procedimento cirúrgico; abordagem do paciente receptor na unidade de internação; preparação para recepção do paciente receptor; realização do procedimento cirúrgico e dos cuidados de enfermagem para o período pós-operatório imediato na sala de recuperação pós-anestésica.

Participaram deste estudo profissionais de enfermagem que atenderam aos seguintes critérios de inclusão: ser profissional da equipe de enfermagem que participa da equipe de transplante hepático no CC, com no mínimo um mês de experiência no cenário de estudo. Os critérios de exclusão foram: profissionais que no período de coleta de dados estavam afastados por motivo de atestado ou férias.

Todos foram convidados individualmente e pessoalmente. Os que aceitaram participar receberam esclarecimentos sobre os objetivos da pesquisa, riscos e benefícios da sua 
participação, além dos aspectos relacionados ao sigilo e ao anonimato. Todos os que aceitaram o convite assinaram o Termo de Consentimento Livre e Esclarecido (TCLE).

Para coleta de dados, utilizaram-se entrevistas semiestruturadas, gravadas em áudio, transcritas pela pesquisadora principal e posteriormente validadas pelos participantes. A análise de dados foi a de conteúdo, organizada em: pré-análise; exploração do material; e tratamento dos resultados com a inferência e a interpretação ${ }^{7}$.

A análise das entrevistas constituiu um plano que foi apresentado para os participantes, pessoalmente e por e-mail, os quais teceram os comentários que permitiram o diagnóstico das prioridades de temas e a identificação de estratégias de educação para composição de um programa de educação permanente.

A referida pesquisa recebeu aprovação do Comitê de Ética em Pesquisa com Seres Humanos da Universidade Federal de Santa Catarina (UFSC), com o Parecer número 1.960 .236 e o Certificado de Apresentação para Apreciação Ética (CAAE) número 61511416.0.0000.0121.

A fim de respeitar o sigilo e o anonimato dos sujeitos da pesquisa, optou-se por identificá-los pela letra $\mathrm{P}$, seguida de um número sequencial, até completar o número de participantes (P1 a P16).

\section{RESULTADOS}

Participaram do estudo 6 enfermeiros, 7 técnicos de enfermagem, 2 auxiliares de enfermagem 1 instrumentador, totalizando 16 integrantes da equipe de transplante hepático, sendo 13 do sexo feminino e 3 do sexo masculino. Em relação à faixa etária: 3 possuem idades entre 21 e 30 anos; 6 entre 31 e 40 anos; 2 entre 41 e 50 anos; 4 entre 51 e 60 anos; e 1 com mais de 61 anos.

Quanto ao tempo que atuam na equipe de transplante, sete estão na equipe há um ano; quatro estão de um ano e um mês a quatro anos; e cinco participam do transplante desde a abertura do serviço, ou seja, há seis anos.

Quanto ao nível de escolaridade, 12 concluíram curso superior, sendo 7 com formação na área da saúde; 4 possuem nível médio (técnico de enfermagem); 3 possuem mestrado; 7 possuem especialização; 2 estão cursando mestrado e 1 está cursando graduação em área diferente da saúde.

A partir dos dados analisados, emergiram três categorias para discussão: conhecimento de todo o processo de transplante hepático; apropriação dos cuidados de enfermagem perioperatória em transplante hepático; e integração e qualificação da equipe interdisciplinar.

\section{Conhecimento de todo o processo de transplante hepático}

Destaca-se a necessidade de conhecimentos e aproximação da equipe com todo o processo de transplante hepático, enquanto ferramenta essencial para a integralidade das ações e dos cuidados desenvolvidos, conforme expresso pelos participantes.

É necessário conhecer o todo... o histórico do paciente, a causa para necessidade de transplante. Durante a cirurgia, estar atento à hemodinâmica do paciente, sinais de sangramento intenso, deve registrar o início da cirurgia, horário do clampeamento, tempo de isquemia até o implante do novo fígado, resultados de exames laboratoriais, além da origem do fígado novo (P11).

É necessário conhecer todo o processo de doação de órgãos e logística do transplante (P12).

Os participantes do estudo reconhecem e compreendem o contexto do transplante, mas sentem necessidade de aprofundamento na logística, processo de doação e captação de órgãos e intervenção cirúrgica do transplante em si para o sucesso dos procedimentos. Observa-se que a implantação de capacitações para todas as categorias da enfermagem é uma decisão assertiva, que os profissionais carecem de atualizações para seu conhecimento científico e que estão bastante receptivos para futuros treinamentos.

\section{Apropriação dos cuidados de enfermagem perioperatória em transplante hepático}

Os participantes apontam para a necessidade de a equipe de enfermagem ser qualificada para exercer o seu cuidado, identificando e estabelecendo prioridades durante o transplante hepático e a atuação perante as intercorrências e aspectos relacionados à sistematização da assistência de enfermagem, abordando, inclusive, os diagnósticos de enfermagem. Incluem, ainda, o manejo de novas tecnologias para a apropriação desse cuidado e sugerem estratégias para melhoria na qualificação profissional.

É necessário participar de simpósios, Jornada Brasileira de transplante. Cursos sobre cuidados de enfermagem pré, intra e pós-transplante, diagnósticos de enfermagem (P5). 
Tivemos a capacitação da máquina de autotransfusão, a qual fica como responsabilidade do enfermeiro, o que facilitou muito o manuseio da mesma durante os transplantes (P15).

Para a apropriação desse cuidado destacam também a necessidade de conhecimento do histórico de saúde-doença do paciente que está sendo submetido ao procedimento. Informações pertinentes ao paciente, tais como: sexo, idade, doenças prévias e de base (P7).

Temos que ter informações, capacitações, cursos, palestras, seminários, conhecimento sobre o assunto que estamos desenvolvendo junto com a equipe. Conhecer outros hospitais, outros serviços para nos qualificar. Oferecer cursos técnicos, graduações para os funcionários. Esclarecer começo, meio e fim do procedimento, ter reuniões para fazer um balanço sobre o trabalho, sobre os pacientes, acidentes (P9).

Os participantes do estudo indicaram a necessidade de ampliar o conhecimento sobre o processo de doação e transplante; comentaram sobre a forma que é incluída na equipe e como ocorre o processo educacional, quanto às necessidades dos pesquisados, e sugeriram propostas educacionais voltadas à qualificação dos profissionais para uma assistência segura e de qualidade.

\section{Integração e qualificação da equipe interdisciplinar}

Os participantes reforçam a relevância da atuação da equipe interdisciplinar para o sucesso e a segurança do processo de transplante hepático. Para efetivação desse cuidado seguro e qualificado, a qualificação dos integrantes é premissa essencial destacada por todos os participantes deste estudo, representados pelas falas a seguir.

Vejo minha atuação como profissional, no transplante hepático; uma equipe funciona cada qual com sua importância, se não estiver integrado, fica dificultoso. Eles devem ser harmônicos e bem treinados, seguros dos seus papéis, em constante evolução (P8).

Com certeza a educação do transplante tem que melhorar, pois o que nós aprendemos foi ensinado por outros colegas, não fizemos cursos, nem capacitações, nada. Aprendemos na prática (P2).

Todo profissional que participa do transplante hepático deveria passar por um treinamento específico para o procedimento, que inclui o preparo do paciente, ato cirúrgico e os tempos cirúrgicos (P4).

Os entrevistados ressaltam, ainda, que essas capacitações devem ter uma continuidade e estar em consonância com as necessidades da equipe.

Creio que encontros, conversas e capacitações devem ser realizados rotineiramente, mesmo para quem já é experiente na equipe, pois sempre podemos aprender coisas novas para aprimorar nosso serviço, mas principalmente para quem inicia, para poder entender melhor o serviço (P6).

Nesse processo, a equipe de enfermagem destaca o enfermeiro enquanto elo entre toda a equipe, articulando-a, orientando, supervisionando, resolvendo contratempos, auxiliando todos enquanto necessário. Por fim, o enfermeiro favorece a integração e a segurança do procedimento, tanto para a equipe quanto para o paciente.

A atuação do enfermeiro é muito importante, somos nós que temos o primeiro contato com o paciente e familiar ainda na unidade e orientamos sobre a cirurgia. Durante a cirurgia devemos perceber toda a movimentação na sala para resolver as intercorrências que vão surgindo e organizar a equipe (P11).

O papel do enfermeiro é fundamental dentro da equipe multiprofissional, pois ele atua na prevenção, tratamento e reabilitação do paciente transplantado, atentando para o bem-estar físico e emocional do paciente, além de fazer um elo entre paciente, família e demais profissionais da saúde. Isso, feito de forma humanizada, proporciona ao paciente e a seus familiares, em um momento de fragilidade, sentirem-se amparados e protegidos (P6).

Os profissionais que atuam no transplante, todos são importantes, mas vejo que o papel do enfermeiro em todo processo é voltado para divulgar, 
desmistificar o tema transplante, sendo de grande valia o elo entre equipe cirúrgica, enfermagem, familiar, doador e receptor (P12).

O intercâmbio entre as várias experiências exitosas foi apontado por alguns como estratégia de educação permanente para o intercâmbio de conhecimentos e vivências na área.

Vivenciar e aprender sobre e com outras realidades e instituições que também realizam transplantes através de palestras, cursos, treinamentos e simulações (P15).

Acho que o hospital poderia ter nos encaminhado para outros centros transplantadores, para entendermos como funcionava. Vejo que no hospital, eles não se preocuparam com os recursos humanos. Até a parte da anestesia deveria ter tido um treinamento $(\mathrm{P} 1)$.

\section{DISCUSSÃO}

A qualificação dos profissionais para o exercício profissional durante o transplante hepático aparece como essencial, havendo necessidade de incluir programas educativos nos serviços de transplantes, com vistas à troca de conhecimento, experiências e vivências ${ }^{8}$. O que se observa, no entanto, é a existência de vários estudos enfocando os períodos pré e pós -operatórios, tratando de cuidados de enfermagem, criação de protocolos e rotinas, tanto na enfermagem quanto nas áreas interdisciplinares ${ }^{4,9-11}$.

As necessidades dos pacientes são identificadas a partir do conhecimento sobre os cuidados de enfermagem pertinentes ao período que precede o transplante, complicações no transplante e cuidados pós-transplante como a maior delas. Também são necessidades, porém com menor interesse por parte dos pacientes, esclarecimentos sobre as medicações após transplante; funcionamento da lista de espera; indicações e contraindicações para transplante; sistema de distribuição de órgãos e o model for end-stage liver disease (MELD — modelo para doença hepática terminal). Um ensino efetivo no período perioperatório para os pacientes repercute em vários benefícios durante todo o processo, sendo alguns deles: diminuição da permanência hospitalar, redução de analgésicos e aumento da satisfação dos pacientes e familiares ${ }^{12}$.
Os cuidadores de pacientes transplantados centram sua preocupação nos cuidados do período pós-operatório, destacando que o meio de informação ideal para o aprendizado sobre esses aspectos deve incluir consultas com enfermeiro e médicos, leitura de manual e grupos de discussão?.

A qualificação profissional perpassa pela preparação e pelo cumprimento de protocolos de cuidados de enfermagem, que contribuem para a padronização das atividades das equipes de transplantes ${ }^{10}$. Esses protocolos podem contribuir para o registro de informações sobre o histórico de saúde-doença do paciente que está sendo submetido ao procedimento (nome, sexo, idade, história mórbida pregressa e atual, procedência, diagnóstico médico, tipo sanguíneo, peso, nome do familiar que o acompanha, dados da internação em enfermaria e reserva de leito na unidade de terapia intensiva - UTI). Os cuidados de enfermagem para pacientes transplantados internados em uma UTI restringiam-se ao cumprimento da prescrição médica, não havendo uma prescrição sobre os seus cuidados ${ }^{11}$. Nesse sentido, recomendaram a implementação da sistematização da assistência de enfermagem (SAE) e a criação/utilização de protocolos de cuidados de enfermagem para pacientes transplantados internados em uma UTI, com vistas a contribuir para a qualidade da assistência de enfermagem ${ }^{10}$.

O aperfeiçoamento e o entendimento de toda a logística do processo de doação de órgãos até o transplante foram identificados pelos participantes do estudo como uma necessidade de aprofundamento, para melhoria na qualidade de assistência, proporcionando base sólida, fazendo com que os novos conhecimentos, as práticas e atitudes sejam eficientes e seguras, transformando-os em profissionais qualificados ${ }^{13}$.

Nesse contexto, o trabalho interdisciplinar favorece a continuidade da assistência e o atendimento ao princípio da integralidade. Sendo assim, diferentes profissionais compartilham seu conhecimento e prestam atendimento integral ${ }^{14,15}$. Ressalta-se que a equipe multidisciplinar realiza, também, as orientações de cuidados após a alta e faz o acompanhamento ambulatorial, minimizando os riscos de efeitos adversos e danos ao paciente, frente às novas medicações utilizadas, prevenindo e promovendo maior segurança ao paciente ${ }^{16}$. O trabalho interdisciplinar é percebido como necessário pelos participantes desta pesquisa, bem como o fato de que todos sejam continuamente capacitados para o transplante hepático.

É fundamental o desenvolvimento de programas de capacitação para os serviços de transplantes. Ainda confirmam que o papel desempenhado pelo enfermeiro é determinante frente à equipe multiprofissional, sendo referência na organização 
de toda estrutura e processo ${ }^{17}$. O enfermeiro é o profissional de conexão entre os profissionais, pacientes e familiares e a implementação de protocolos para cuidado da enfermagem é uma tecnologia que traduz segurança e eficácia na atuação dos profissionais. O enfermeiro foi identificado por toda a equipe como membro essencial de todo o processo, sendo ele o profissional que planeja, coordena, gerencia e organiza toda a logística para que o transplante hepático possa ser bemsucedido em suas várias etapas. Neste sentido, o enfermeiro é o elo entre sua equipe, o paciente e a família, promovendo a realização do cuidado de enfermagem com qualidade, assim como é o profissional que trabalha na gestão do cuidado, promovendo a educação continuada e a melhoria da qualidade do atendimento, envolvendo aspectos éticos e sociais ${ }^{12,16}$. As limitações impostas pelas realidades dos centros transplantadores, o número reduzido de profissionais, de materiais e recursos financeiros limitam a dinâmica do enfermeiro em prestar um atendimento seguro e de qualidade ${ }^{12}$.

No contexto nacional, a Portaria $\mathrm{n}^{\circ} 356$, de 10 de março de 2014, do Ministério da Saúde, publica boas práticas em procedimentos para organização e funcionamento dos serviços de transplante de órgãos, incluindo a capacitação de todos os membros da equipe e um trabalho interdisciplinar, ou seja, uma equipe, com várias especialidades, envolvida em um único foco, o paciente; buscando trabalho com segurança $^{18}$.

A implantação da educação permanente é uma boa estratégia para qualificar os profissionais, pois todos os envolvidos analisam criticamente suas atividades, destacando os problemas e desenvolvendo melhor compreensão e valorização da prática, com a normatização da assistência. Neste sentido, a prática é a fonte do conhecimento científico, entendendo que a educação permanente deve fazer parte do contexto profissional de saúde, pois fornece o empoderamento para transformar os problemas em ferramentas para melhoria do cuidado $^{19,20}$. Paralelamente à educação em serviço, a padronização da assistência de enfermagem, por meio de uma construção coletiva e de pesquisas, torna o exercício profissional seguro ${ }^{21}$.

Assim, a criação de novas tecnologias para normatização do cuidado, ou seja, a criação de padronização por meio de procedimento operacional padrão $(\mathrm{POP})$ e a organização e implementação da SAE, voltada para o período intraoperatório, geram impacto no processo de trabalho, estruturando a atividade e harmonizando o serviço. Essas tecnologias fazem com que a prática se torne mais eficaz e mais eficiente, gerando um controle sobre a prática assistencial.
Também emergem das falas outras estratégias, tais como: implementação da semana de transplante hepático na instituição, com a organização de cursos e palestras; visitas técnicas às instituições que realizam transplante hepático, presenciais ou por videoconferência, com temas que se voltem para a interação entre as equipes de transplante, interação interpessoal, realização de estágios em outros centros; capacitações interdisciplinares e contínuas; elaboração de manuais para consulta em serviço; cursos técnicos para a equipe de enfermagem; participação em eventos (congressos e jornadas de transplantes; simpósio de especialistas em imunossupressão), bem como a criação de uma agenda de reuniões com a participação de todos os profissionais.

As estratégias elencadas demandam um esforço de cooperação entre os profissionais da equipe de transplante e a instituição, pois dependem de uma reorganização do serviço. Por essa razão, destaca-se a necessidade de que ocorra uma mudança institucional no sentido de que se promova a formação de profissionais, qualificando-os por meio de capacitações permanentes a partir das necessidades do serviço reveladas pela equipe de trabalho ${ }^{22}$.

\section{CONSIDERAÇÕES FINAIS}

A prática de enfermagem impulsiona métodos facilitadores para o cuidado; e a implementação de atividades educativas no cotidiano do profissional projeta o saber-fazer de uma forma sistematizada, científica e de qualidade, padronizando as condutas de enfermagem, proporcionando maior segurança nas ações executadas. A proposta educativa serve como ferramenta para auxiliar no processo ensino-aprendizagem e nas pesquisas científicas.

A limitação encontrada foi a escassez de produções, na área de enfermagem, de materiais educacionais para os profissionais que atuam no período intraoperatório no transplante hepático.

Devido à complexidade do tema, deseja-se que esta pesquisa sirva de auxílio para outros centros transplantadores, sendo significativo o aprofundamento no que concerne à implantação do serviço com embasamento em um referencial metodológico para alicerçar a prática, assim configurando-o como um instrumento científico para o desenvolvimento das atividades e a formação de programas de educação permanente, de acordo com as necessidades das equipes que atuam com essa realidade. 


\section{REFERÊNCIAS}

1. Brasil. Ministério da Saúde. Secretaria de Gestão do Trabalho e da Educação na Saúde. Departamento de Gestão da Educação em Saúde. Política Nacional de Educação Permanente em Saúde. Brasília: Ministério da Saúde; 2009. 64p.

2. Baldissera VDA, Bueno SMV. A educação permanente em saúde e a educação libertária de Paulo Freire. Ciênc Cuid Saúde. 2014 Abr/Jun;13(2):191-2. http://dx.doi.org/10.4025/cienccuidsaude. v13i2.26545

3. Jesus MCP, Figueiredo MAG, Santos SMR, Amaral AMM, Rocha LO, Thiollent MJM. Permanent education in nursing in a university hospital. Rev Esc Enferm USP [Internet]. 2011 Out [citado em 10 abr. 2018];45(5):1229-36. Disponível em: http://dx.doi.org/10.1590/ S0080-62342011000500028

4. Mendes KDS, Roza BA, BarbosaSFF, Schirmer J, Galvão CM. Transplante de órgãos e tecidos: responsabilidades do enfermeiro. Texto Contexto Enferm [Internet]. 2012 Dez [citado em 10 abr. 2018];21(4):945-53. Disponível em: http://dx.doi.org/10.1590/S0104-07072012000400027

5. Polit DF, Beck CT. Fundamentos de pesquisa em enfermagem: avaliação de evidência para a prática da enfermagem. $7^{a}$ ed. Porto Alegre: Artmed; 2011.

6. Brasil. Relatórios anuais transplante equipe Hospital Universitário de Santa Catarina, 2017. Brasília; 2017. [citado em 17 mar. 2018]. Disponivel em: https://snt.saude.gov.br

7. Minayo MCS. 0 desafio do conhecimento: pesquisa qualitativa em saúde. $14^{\mathrm{a}}$ ed. São Paulo: Hucitec; 2014.

8. Morais TR, Morais MR. Doação de órgãos: é preciso educar para avançar. Saúde Debate [Internet]. 2012 Dez [citado 10 abr. 2018];36(95):633-9. Disponivel em: http://dx.doi.org/10.1590/S0103-11042012000400015

9. Mendes KDS, Rossin FM, Ziviani LC, Castro-e-Silva O, Galvão CM. Necessidades de informação de candidatos ao transplante de fígado: o primeiro passo do processo ensino-aprendizagem. Rev Gaúcha Enferm [Internet]. 2012 Dez [citado em 10 abr. 2018];33(4):94-102. Disponível em: http://dx.doi.org/10.1590/S1983-14472012000400012

10. Marandola PG, Matos SS, De Mattia AL, Rocha ADM, Silva JS, Resende MKB. Consulta de enfermagem ao paciente em pré-transplante de fígado: elaboração de um protocolo. Rev Enferm Centro Oeste Mineiro. 2011;1(3):324-31. http://dx.doi.org/10.19175/recom.v0i0.62

11. Borges MCLA, Silva LMS, Guedes MVC, Caetano JA. Desvelando o cuidado de enfermagem ao paciente transplantado hepático em uma Unidade de Terapia Intensiva. Esc Anna Nery [Internet]. 2012 Dez [citado em 10 abr. 2018];16(4):754-60. Disponível em: http:// dx.doi.org/10.1590/S1414-81452012000400016

12. Silva AS, Valácio RA, Botelho FC, Amaral CFS. Fatores de atraso na alta hospitalar em hospitais de ensino. Rev Saúde Pública [Internet] 2014 Jun [citado em 10 abr. 2018];48(2):314-321. Disponível em: http://www.scielo.br/pdf/rsp/v48n2/0034-8910-rsp-48-2-0314.pdf
13. Silva OC, Souza FF, Teixeira AC, Mente ED, Sankarankutty AK. Transplante de fígado em nosso meio: a evolução de um procedimento estratégico institucional. Medicina Ribeirão Preto. 2009;42(4):482-4. http://dx.doi.org/10.11606/issn.2176-7262.v42i4p482-484

14. Bispo EPF, Tavares CHF, Tomaz JMT. Interdisciplinaridade no ensino em saúde: o olhar do preceptor na Saúde da Família. Interface [Internet]. 2014 Jun [citado em 10 abr. 2018];18(49):337-50. Disponível em: http://dx.doi.org/10.1590/1807-57622013.0158

15. Alvarenga JPA, Meira AB, Fontes WD, Xavier MMFB, Trajano FMP, Chaves Neto G, et al. Multiprofessional and interdisciplinary in health education: experiences of undergraduates on regional interdisciplinary internship. J Nurs UFPE. 2013;7(10):5944-51. https:// doi.org/10.5205/1981-8963-v7i10a12221p5944-5951-2013

16. Lima LF, Martins BCC, Oliveira FRP, Cavalcante RMA, Magalhães VP, Firmino PYM, et al. Orientação farmacêutica na alta hospitalar de pacientes transplantados: estratégia para a segurança do paciente. Einstein [Internet]. 2016 [citado em 10 abr. 2018];14(3):359-65. Disponível em: http://dx.doi.org/10.1590/S1679-45082016A03481

17. Negreiros FDS, Marinho AMCP, Garcia JHP, Morais APP, Aguiar MIF, Carvalho SL. Captação do fígado do doador para o transplante: uma proposta de protocolo para o enfermeiro. Esc Anna Nery. 2016;20(1):38-47. http://dx.doi.org/10.5935/1414-8145.20160006

18. Brasil. Ministério da Saúde. Portaria n. 356, de 10 de março de 2014. Publica a proposta de Projeto de Resolução "Boas Práticas em procedimentos para organização e funcionamento dos serviços de transplante de órgãos" [Internet]. Brasília: Ministério da Saúde; 19 out 2014 [citado em 10 abr. 2018]. Disponível em: http://bvsms.saude.gov.br/bvs/saudelegis/ gm/2014/prt0356_10_03_2014.html

19. Brasil. Ministério da Saúde. Portaria n ${ }^{\circ} 2.600$, de 21 de outubro de 2009. Aprova o Regulamento Técnico do Sistema Nacional de Transplantes [Internet]. Brasília: Ministério da Saúde; 21 out 2009 [citado em 10 abr. 2018]. Disponível em: http://www.ans.gov.br/images/stories/ Legislacao/camara_tecnica/2013_gt_revisao_rol/20130520-doc12 contribconsnacdesauderegulamentotecnicodosnt.pdf

20. Magalhães ALP. Gerenciando o cuidado de enfermagem no processo de doação e transplantes de órgãos e tecidos na perspectiva do pensamento Lean [doutorado]. Florianópolis: Universidade Federal de Santa Catarina; 2015.

21. Conselho Regional de Enfermagem de São Paulo. Guia para a construção de protocolos assistenciais de enfermagem [Internet]. 2014 [citado em 10 nov. 2016]. Disponível em: http://portal.corensp. gov.br/sites/default/files/guia\%20constru\%C3\%A7\%C3\%A30\%20 protocolos\%2025.02.14.pdf

22. Arcanjo RA, Oliveira LC, Silva DD. Reflexões sobre a comissão intra-hospitalar de doação de órgãos e tecidos para transplantes. Rev Bioética. 2013;21(1):119-25. http://dx.doi.org/10.1590/ S1983-80422013000100014 